\title{
Factors Associated With the Use of Meals-0n- Wheels among Home Care Clients: The Nutormed Study
}

\author{
Irma Nykänen ${ }^{1,2 *}$, Miia Tiihonen ${ }^{1}$, Prof Sirpa Hartikainen ${ }^{1}$ \\ ${ }^{1}$ Kuopio Research Centre of Geriatric Care, School of Pharmacy, Faculty of Health Sciences, University of Eastern Finland, Kuopio, Finland \\ ${ }_{2}^{2}$ Institute of Public Health and Clinical Nutrition, Unit of Public Health, Faculty of Health Sciences, University of Eastern Finland, Kuopio, Finland
}

Received: September 22, 2016; Accepted: October 31, 2016; Published: November 07, 2016

*Corresponding author: Irma Nykänen, Kuopio Research Centre of Geriatric Care, School of Pharmacy /Institute of Public Health and Clinical Nutrition, Unit of Public Health, Faculty of Health Sciences, University of Eastern Finland, Kuopio, Finland. Tel. No: +35840 3552991; Fax: +35817162131; E-mail: Irma.Nykanen@uef.fi

\begin{abstract}
Background: The risk of malnutrition is quite common among home care clients. To aim of this study were to describe nutritional status of Meals-on-Wheels (MOW) clients and factors associated with use of MOW among home care clients.

Methods: This cross-sectional study was conducted as part of the Nutrition, Oral health and Medication (NutOrMed) study. The study population consisted of 266 home care clients in the year 2013, MOW clients $(n=153)$ and non-MOW clients $(n=113)$. The data contained information on socio demographic factors, nutritional status, functional ability, depressive symptoms, comorbidities, dementia diagnosis and drug use.
\end{abstract}

Results: The mean (SD) age of the home care clients was 84.4 (5.3) years and $73 \%(n=196)$ of them were female. $90 \%(n=139)$ of MOW clients were at risk of malnutrition or malnourished, compared to $80 \%(n=90)$ of non-MOW clients. MOW clients were more likely to be living alone, impairments in the activities of daily living (ADL) and have diagnosis of a dementive disorder. Clients who were unsatisfied with MOW were more likely to be at risk of malnutrition or malnutrition and depressive (GDS-15).

Conclusion: Using MOW is common among home care clients and MOW clients were more likely to be at risk of malnutrition, living alone, have a decline in the performance of activities of daily living and diagnosed dementia. Unsatisfied with the quality of MOW were more likely to be at risk of malnutrition or malnourished and depressive. People:

Keywords: Home Care Clients; Meals-on-Wheels; MNA; Older

\section{Introduction}

Meals-on-Wheels (MOW) is an ancillary service that deliver meals to individuals at home who are unable to purchase or prepare their own meals. This is taking place in Finland and nowadays about $25 \%$ of Finns aged above 80 years use MOW [1]. MOW helps home care clients maintain and improve their nutritional status and may help increase older peoples' independence while encouraging autonomy [2]. Using MOW services improves older people's nutritional intake [3, 4]. A previous study among community-dwelling older people showed that use of coupled with individual nutritional counselling can improve nutritional status [5]. The main ideas of MOW are to provide not only a portion of the daily required nutritional intake $[4,7]$, but also meals that clients can consume fully [8].

Nowadays, the objective is to facilitate living at home as long as possible and to provide home care services. To provide adequate treatment and care according also to the individual needs of these home care clients, health care and nursing care providers need to know more about this group. It is important to identify MOW clients among the vulnerable population so that the service can be improved. We also need understand better how MOW are used. The aims of this study were to describe nutritional status of MOW clients and factors associated with use of.

\section{Methods}

This cross-sectional study was conducted as part of the Nutrition, Oral health and Medication (NutOrMed) study [9]. The study sample comprised $(n=266)$ home care clients aged 75 years or over living in three cities in Eastern and Central Finland. Home care clients were interviewed and examined at home by a nutritionist, trained nurses and a pharmacist between February and December 2013. If the participant had a cognitive impairment, the data were supplemented by a caregiver.

Nutritional status and meal service usage were evaluated by a nutritionist. She measured weight and height and computed BMI as the ratio of weight to the square of height $\left(\mathrm{kg} / \mathrm{m}^{2}\right)$. Nutritional screening was performed using the Mini Nutritional Assessment (MNA) [10]. Meal service assessment included questions about how many times MOW were used per week. The participants who received MOW $\geq 3$ times per week were included in MOW clients. 
The quality of MOW was assessed on a 3-point scale: $1=$ good quality, 2 = acceptable quality, 3 = poor quality. Plasma albumin levels were measured according to standard protocols at the regional laboratory, ISLAB [11]. Body Mass Index (BMI) was computed as the ratio of weight to the square of height $\left(\mathrm{kg} / \mathrm{m}^{2}\right)$.

Performance in Activities of Daily Living (ADL) was assessed with the 10-item Barthel ADL Index [12] and performance in instrumental activity (IADL) [13], with the 8-item Lawton and Brody Scale. The scoring for ADL is $0-100$ and for IADL, $0-8$, with higher scores indicating better functioning. Depressive symptoms were evaluated on the 15-item Geriatric Depression Scale (GDS-15) [14]. Comorbidity was computed using a modified version of the Functional Comorbidity Index (FCI) [15]. Diagnosis of 13 diseases were collected from electronic medical records by a geriatrician. Each condition was given one point, and a higher FCI sum score represents greater comorbidity. Also dementia diagnosis was collected by a geriatrician and was based on electronic medical records of health care. A pharmacist interviewed the participants at the baseline and recorded each prescription and over-the-counter drug used regularly and as needed on the basis of the interview, medication lists and medication packages.

In statistical analyses the participants were categorized into users and non-users of MOW (Table 1). Statistical comparisons between the groups were done using a chi-square or t-test with $\leq 0.05$ considered significant. Univariate and multivariate (stepwise, forward selection) regression analyses were performed to identify demographical, clinical and functional factors associated with using MOW (Table 2). The MOW users were categorized into good and weak of quality of MOW and statistical comparisons between the groups were done using a chi-square or t-test (Table 3). The analyses were done using SPSS version 21.0 (SPSS, Inc., Chicago, IL).

All the participants or their proxies gave written informed consent to participate in the study. The study protocol was approved by the Research Ethics Committee of the Northern Savo Hospital District, Kuopio, Finland.

\section{Results}

The mean (SD) age of the home care clients was 84.4 (5.3) years and $73 \%(n=196)$ of them were female. In the group of MOW clients, $90 \%(n=139)$ were at risk of malnutrition or malnourished, compared to $80 \%(n=90)$ of non-MOW clients (Table 1). In the multivariate analysis, MOW clients 77 were more likely to be living alone $(\mathrm{OR}=5.20,95 \%$ confidence interval CI: 2.54-10.80), impairments in the Activities of Daily Living (ADL) (OR $=1.11,95 \% \mathrm{CI}: 1.02-1.19$ ) and diagnosis of a dementive disorder (OR $=2.38,95 \% \mathrm{CI}: 1.33-4.25)$ (Table 2). In the group of unsatisfied quality of MOW, $94.5 \%(n=86)$ were at risk of malnutrition or malnourished and $57 \%(n=51)$ have depressive symptoms, compared to $50 \%(n=31), 34 \%(n=21)$ respectively of satisfied the quality of MOW (Table 3).

\section{Discussion}

Home care clients receiving MOW were more frequently at risk of malnutrition or malnutrition, lived alone, had decline in activities of daily living and were demented. Also in an earlier cross-sectional study among MOW clients, the prevalence of malnutrition risk or malnutrition was higher than non-MOW clients [16]. The reason for MOW client's poorer nutritional status is unclear according to our study because we did not have earlier information about them. However, in one Swedish study the participants who used MOW were more likely to be at risk of malnutrition [17]. It is reasonable to assume that malnutrition leads to increased need for help from the community, including meal services.

Table 1: Characteristics of clients and non-clients of Meals on Wheels (MOW)

\begin{tabular}{|c|c|c|c|}
\hline & $\begin{array}{c}\text { MOW } \\
\mathbf{n = 1 5 3}(\mathbf{5 7 . 7})\end{array}$ & $\begin{array}{r}\text { non-MOW } \\
\mathbf{n = 1 1 3}(\mathbf{4 2 . 3})\end{array}$ & P value \\
\hline Women, n (\%) & $119(77.3)$ & $77(68.1)$ & 0.095 \\
\hline Age, mean \pm SD & $84.7 \pm 5.2$ & $83.9 \pm 5.6$ & 0.244 \\
\hline Living alone, n (\%) & $109(73.6)$ & $59(53.6)$ & 0.001 \\
\hline MNA <24.0, n (\%) & $139(90.3)$ & $90(79.6)$ & 0.014 \\
\hline BMI, mean \pm SD & $27.3 \pm 6.4$ & $27.0 \pm 4.2$ & 0.635 \\
\hline P-alb, mean \pm SD & $36.3 \pm 3.4$ & $36.9 \pm 4.0$ & 0.341 \\
\hline ADL Barthel Index, mean & $80.9 \pm 20.6$ & $87.0 \pm 17.4$ & 0.012 \\
\hline IADL, SD & $4.1 \pm 2.1$ & $5.2 \pm 2.5$ & 0.001 \\
\hline $\begin{array}{c}\text { GDS-15 } \geq 5, \mathrm{n}(\%) \\
\text { FCI, mean } \pm \text { SD }\end{array}$ & $71(47.4)$ & $41(38.0)$ & 0.122 \\
\hline $\begin{array}{c}\text { Dementia diagnosis, n } \\
(\%)\end{array}$ & $72(46.8)$ & $38(34.2)$ & 0.041 \\
\hline $\begin{array}{c}\text { Excessive polypharmacy, } \\
\text { n (\%) }\end{array}$ & $133(88.1)$ & $98(88.3)$ & 0.959 \\
\hline
\end{tabular}

SD, Standard Deviation; MNA, Mini Nutritional Assessment; BMI, Body Mass Index; P-alb, Plasma albumin; ADL, activities of daily living; IADL, Instrumental Activities of Daily Living; GDS-15, Geriatric Depression Scale-15; FCI, Functional Comorbidity Index

Table 2: Univariate and multivariate associations between characteristics of home care clients and using of Meals on Wheels

\begin{tabular}{|c|c|c|}
\hline & $\begin{array}{c}\text { Univariate } \\
\text { OR (95\% CI) }\end{array}$ & $\begin{array}{c}\text { Multivariate }^{\mathbf{a}} \\
\text { OR (95\% CI) }\end{array}$ \\
\hline Sex (female) & $1.60(0.92-2.76)$ & \\
\hline Age & $1.02(0.98-1.07)$ & \\
\hline Living alone & $2.43(1.44-4.11)$ & $3.37(1.88-6.05)$ \\
\hline MNA, $<24$ & $2.38(1.18-4.81)$ & \\
\hline ADL Barthel Index & $1.08(1.02-1.16)$ & $1.11(1.02-1.19)$ \\
\hline GDS-15, $\geq 5$ & $1.48(0.89-2.46)$ & \\
\hline FCI & $1.26(1.09-1.47)$ & \\
\hline Dementia diagnosis & $1.68(1.02-2.79)$ & $2.38(1.33-4.25)$ \\
\hline $\begin{array}{c}\text { Excessive } \\
\text { polypharmacy }\end{array}$ & $1.02(0.47-2.18)$ & \\
\hline
\end{tabular}

a: Forward Wald selection. Only variables that entered the model are shown.

OR, odds ratio; MNA, ADL, activities of daily living; Mini Nutritional Assessment; FCI, Functional Comorbidy Index; GDS-15, Geriatric Depression Scale 
In accordance with previous studies, MOW clients are more likely to be aged 75 or older, female, living alone, less mobile and widowed than non-MOW clients [18-21]. Older women living alone tended to simplify cooking and eating and had fewer cooked meals, whereas among older men, poor cooking skills were a barrier to maintaining healthy eating and a good appetite [22]. It is reasonable to suggest that older people living alone eat more poorly, which affects their nutritional status [18], and this results in them using MOW. Many of the MOW clients were at a low level of economic resources, so meals were often split into two. Perhaps MOW clients split their delivered, midday meal between lunch and dinner because they had poor appetite or if portion size of meal was too big to eat all at once.

We found that clients of MOW with dementia and declining performance in activities of daily living were unable to or had difficulty preparing meals on their own. This is in accordance with previous findings that persons with a cognitive and/or functional impairment are unable to prepare nutritionally adequate meals [22]. In addition, at a later stage of dementia, persons have difficulty recognizing hunger and thirst and may forget to eat, or they are unable to recognize items of food [23].

In the present study, the clients of unsatisfied of MOW were at risk of malnutrition or malnourished and depressive more often than the clients of satisfied of MOW. Earlier studies have documented rates of depression among older adults currently receiving in-home services [24]. Lack of appetite, loss of interest in self-care, apathy and physical weakness can be used early warning signals not only the risk of malnutrition or malnutrition, also depression [25].

Table 3: Characteristics of clients of Meals on Wheels (MOW) based on the quality of MOW

\begin{tabular}{|c|c|c|c|}
\hline & $\begin{array}{c}\text { Good } \\
n=62(40.4)\end{array}$ & $\begin{array}{c}\text { Weak } \\
\mathrm{n}=91(59.6)\end{array}$ & $P$ value \\
\hline Women, n (\%) & $46(74.2)$ & $72(79.1)$ & 0.476 \\
\hline Age, mean \pm SD & $85.1 \pm 5.1$ & $84.5 \pm 5.2$ & 0.470 \\
\hline MNA < 24.0, n (\%) & $31(50.0)$ & $86(94.5)$ & 0.001 \\
\hline BMI, mean \pm SD & $27.1 \pm 5.8$ & $27.5 \pm 6.8$ & 0.715 \\
\hline P-alb, mean \pm SD & $36.2 \pm 3.3$ & $36.6 \pm 3.4)$ & 0.886 \\
\hline $\begin{array}{l}\text { ADL, Barthel Index, } \\
\text { mean } \pm \text { SD }\end{array}$ & $83.4 \pm 16.7$ & $79.0 \pm 22.9$ & 0.173 \\
\hline $\mathrm{IADL}$, mean $\pm \mathrm{SD}$ & $4.2 \pm 2.0$ & $4.2 \pm 2.2$ & 0.965 \\
\hline GDS-15, > 5, n (\%) & 21 (33.9) & $51(57.3)$ & 0.040 \\
\hline MMSE, mean \pm SD & $23.0 \pm 4.4$ & $21.9 \pm 5.5$ & 0.191 \\
\hline $\mathrm{FCI}$, mean $\pm \mathrm{SD}$ & $3.4 \pm 1.9$ & $3.1 \pm 2.0$ & 0.338 \\
\hline $\begin{array}{c}\text { Dementia diagnosis, } \\
n(\%)\end{array}$ & 32 (51.6) & $39(43.3)$ & 0.315 \\
\hline $\begin{array}{c}\text { Excessive } \\
\text { polypharmacy, } \mathrm{n}(\%)\end{array}$ & $41(41.1)$ & 38 (58.9) & 0.384 \\
\hline \multicolumn{4}{|c|}{$\begin{array}{l}\text { SD, Standard Deviation; MNA, Mini Nutritional Assessment; BMI, Body } \\
\text { Mass Index; P-alb, Plasma albumin; ADL, activities of daily living; IADL, } \\
\text { Instrumental Activities of Daily Living; GDS-15, Geriatric Depression } \\
\text { Scale-15; FCI, Functional Comorbidity Index }\end{array}$} \\
\hline
\end{tabular}

Our study results are especially important now, when the number of home care clients is rapidly growing because of the decrease in residential care. In addition, the prevalence of malnutrition or risk of malnutrition is very high among this vulnerable population. The MOW service is based on the assumption that all of the food is actually consumed, which leads to an overly positive picture of the nutritional value of the meals. Looking to the future, it is essential that MOW is able to adapt to consumer needs and preferences and respond to challenges in MOW production and delivery.

\section{Conclusion}

Using MOW is common among home care clients and MOW clients were more likely to be at risk of malnutrition, living alone, have a decline in the performance of activities of daily living and diagnosed dementia. Unsatisfied with the quality of MOW were more likely to be at risk of malnutrition or malnourished and depressive.

\section{Acknowledgements}

We would like to acknowledge the staff included in this study for their positive attitude during the data collection.

\section{Declarations}

The authors confirm that they have no conflict of interest. University of Eastern Finland and North Savo Regional Fund have funded this study. Irma Nykänen, PhD. prepared the manuscript. All authors critically reviewed the manuscript and approved the final version submitted for publication.

\section{Trial registration}

\section{ClinicalTrials.gov: NCT02214758}

\section{Reference}

1. Alden-Nieminen H, Raulio S, Männistö S, Laitalainen E, Suominen M, Prättälä R. Meal patterns among the Finnishelderly. Monitoring project of mass catering. 2009.

2. Sahyoun NR, Vaudin A. Home-Delivered Meals and Nutrition Status Among Older Adults. Nutr Clin Pract. 2014;29(4):459-654.

3. Keller HH. Meal programs improve nutritional risk: a longitudinal analysis of community-living seniors. J Am Diet Assoc. 2006;106(7):1042-1048.

4. Millen BE, Ohls JC, Ponza M, McCool AC. The elderly nutrition program: an effective national framework for preventive nutrition interventions. J Am Diet Assoc. 2002;102(2):234-240.

5. Nykänen I, Rissanen T, Hartikainen S. Meals-on-wheels with individual dietary counseling can improve nutritional status in older people. Journal of Nutritional Health \& Food Science. 2014;2(4):1-4. Doi: doi. org/10.15226/jnhfs.2014.00129

6. Roy MA, Payette H. Meals-On-Wheels improves energy and nutrient intake in a frail free-living elderly population. J Nutr Health Aging. 2006;10(6):554-560.

7. O'Dwyer C, Corish CA, Timonen V. Nutritional status of Irish older people in receipt of meals-on-wheels and the nutritional content of meals provided. J Hum Nutr Diet. 2009;22(6):521-527. doi: 10.1111/j.1365-277X.2009.00992.x. 
8. Krassie J, Smart C, Roberts DC. A review of the nutritional needs of Meals on Wheels consumers and factors associated with the provision of an effective meals on wheels service-an Australian perspective. Eur J Clin Nutr. 2000;54(4):275-280.

9. Tiihonen M,Autonen-Honkonen K, Ahonen R, Komulainen K, Suominen L, Hartikainen S, et al. NutOrMed-optimising nutrition, oral health and medication for older home care clients-study protocol. BMC Nutrition. 2015;1:13. DOI: 10.1186/s40795-015-0009-7

10. Guigoz Y, Vellas B, Garry PJ. Assessing the nutritional status of the elderly: The Mini Nutritional Assessment as part of the geriatric evaluation. Nutr Rev. 1996;54(1 Pt 2):S59-S65.

11. Patient Guide. The web manual of Eastern Finland Laboratory Centre (In Finnish).

12. Mahoney FI, Barthel DW. Functional Evaluation: the Barthel Index. Md State Med J. 1965;14:61-65.

13. Lawton MP, Brody EM. Assessment of older people: selfmaintaining and instrumental activities of daily living. Gerontologist. 1969;9(3):179-186.

14. Sheik J YJ Sheik J, Yesavage J. Geriatric Depression Scale (GDS): recent evidence and 161 development of a shorter version. Clin Gerontol. 1986;5:165-172.

15. Tikkanen P, Nykanen I, Lonnroos E, Sipilä S, Sulkava R, Hartikainen S. Physical activity at age of 20-64 years and mobility and muscle strength in old age: a community-based study. J Gerontol A Biol Sci Med Sci. 2001;67(8):905-910. doi: 10.1093/gerona/gls005

16. Kiesswetter E, Pohlhausen S, Uhlig K, Diekmann R, Lesser S, Heseker $\mathrm{H}$ et al. Malnutrition is related to functional impairment in older adults receiving home care. J Nutr Health Aging. 2013;174(4):345-350. DOI: 10.1007/s12603-012-0409-1
17. Saletti A, Johansson L, Yifter-Lindgren E, Wissing U, Osterberg K, Cederholma T. Nutritional status and a 3-year follow-up in elderly receiving support at home. Gerontology. 2013;51(3):192-198.

18. Ferry M, Sidobre B, Lambertin A, Barberger-Gateau P. The SOLINUT study: analysis of the interaction between nutrition and loneliness in persons aged over 70 years. J Nutr Health Aging. 2005;9(4):261-268.

19. Ramic E, Pranjic N, Batic-Mujanovic O, Karic E, Alibasic E, Alic A. The effect of loneliness on malnutrition in elderly population. Med Arh. 2011;65(2):92-95.

20. Brownie S, Myers SP, Stevens J. The value of the Australian nutrition screening initiative for older australians--results from a national survey. J Nutr Health Aging. 2007;11(1):20-25.

21. Frongillo EA, Wolfe WS. Impact of participation in Home-Delivered Meals on nutrient intake, dietary patterns, and food insecurity of older persons in New York state. J NutrElder. 2010;29(3):293-310. doi: 10.1080/01639366.2010.499094.

22. Coulston AM, Craig L, Voss AC. Meals-on-wheels applicants are a population at risk for poor nutritional status. J Am Diet Assoc. 1996;96(6):570-573.

23. Aselage MB, Amella EJ. An evolutionary analysis of mealtime difficulties in older adults with dementia. J Clin Nurs. 2010;19(1):3341. doi: 10.1111/j.1365-2702.2009.02969.x.

24. Thomas KS, Smego R Akobundu U, Dosa D. Characteristics of Older Adults on Waiting Lists for Meals on Wheels: Identifying Areas for Intervention. J Appl Gerontol. 2005;1-15.

25. Ferdous T, Cederholm T, Razzaque A, Wahlin A, Nahar Kabir Z. Nutritional status and self-reported and performance-based evaluation of physical function of elderly persons in rural Bangladesh. Sc and J Public Health. 2009;37(5):518-524. doi: $10.1177 / 1403494809102778$ 